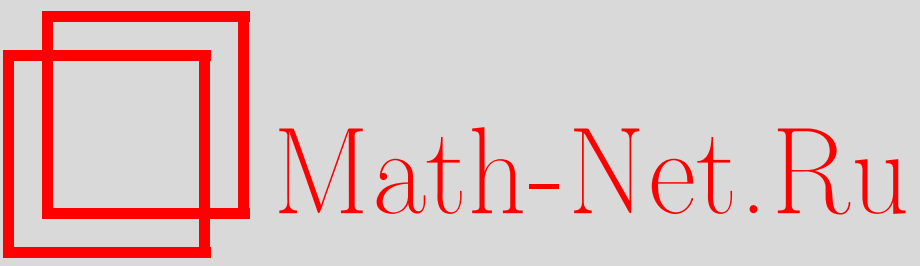

М. А. Ольшанецкий, Построение решений периодической цепочки Тоды с помощью процедуры проектирования и алгебро-геометрическим методом, ТМФ, 2001, том 128, номер 3, 461-473

DOI: https://doi.org/10.4213/tmf509

Использование Общероссийского математического портала Math-Net.Ru подразумевает, что вы прочитали и согласны с пользовательским соглашением

http: //www . mathnet.ru/rus/agreement

Параметры загрузки:

IP : 3.89 .185 .249

26 апреля 2023 г., 13:12:58 


\section{ПОСТРОЕНИЕ РЕШЕНИЙ ПЕРИОДИЧЕСКОЙ ЦЕПОЧКИ ТОДЫ С ПОМОЩЬЮ ПРОЦЕДУРЫ ПРОЕКТИРОВАНИЯ \\ И АЛГЕБРО-ГЕОМЕТРИЧЕСКИМ МЕТОДОМ}

Сравниваются различные способы построения решений периодической цепочки Тоды. Предложены два способа, которые следуют из метода проектирования. Эти способы сравниваются с алгебро-геометрической конструкцией Кричевера.

\section{1. ВВЕДЕНИЕ}

Большой класс конечномерных классических вполне интегрируемых систем может быть получен из свободных систем с помошью симплектической редукции. Этот класс содержит рациональные и тригонометрические модели Калоджеро-Мозера [1]-[3], открытую цепочку Тоды [4], [5], эллиптическую модель Калоджеро-Мозера [6], [7]. Некоторые из этих систем являются частными примерами систем типа Хитчина, которые строятся с помощью симплектической редукции из двумерной свободной гамильтоновой теории поля, определенной на базисных спектральных кривых [8]. Процедура редукции попутно позволяет построить представление Лакса и описать решения задачи Коши методом проектирования.

Метод проектирования заключается в следующем. Пусть $\mathcal{R}$ - исходное фазовое пространство, описывающее свободную иерархию, $\mathcal{R}^{\text {red }}$ - редуцированное фазовое пространство. Это означает, что существует группа калибровочных симметрий $\mathcal{G}$ гамильтоновой иерархии на $\mathcal{R}$. Пространство $\mathcal{R}^{\text {red }}$ определяется в два этапа. Сначала с помощью $\mathcal{G}$ фиксируется калибровка, а затем находятся решения соответствующего закона Гаусса (т.е. уравнения моментов). Множество решений этих уравнений образует $\mathcal{R}^{\text {red }}$. Эта двухэтапная процедура обозначается как $\mathcal{R}^{\mathrm{red}} \sim \mathcal{R} / / \mathcal{G}$.

Для решения задачи Коши начальные данные $x_{0} \in \mathcal{R}^{\text {red }}$ поднимаются в расширенное фазовое пространство $\mathcal{R}\left(X_{0}=\pi^{-1}\left(x_{0}\right)\right)$. Эволюция на $\mathcal{R}$ линейна:

$$
X\left(t_{1}, t_{2}, \ldots\right)=X_{0}+\sum_{j} F_{j}\left(X_{0}\right) t_{j} .
$$

${ }^{*}$ Институт теоретической и экспериментальной физики, Москва, Россия. E-mail: olshanet@heron.itep.ru 
Решение $x\left(t_{1}, t_{2}, \ldots\right)$ на $\mathcal{R}^{\text {red }}$ определяется как калибровочное преобразование $\pi$ (проекция) $X\left(t_{1}, t_{2}, \ldots\right)$ на редуцированное фазовое пространство $\mathcal{R}^{\text {red }}$

$$
x\left(t_{1}, t_{2}, \ldots\right)=\pi\left(X\left(t_{1}, t_{2}, \ldots\right)\right) .
$$

Последний шаг - наиболее трудная часть метода проектирования, однако для некоторых систем она оказывается чрезвычайно простой. В частности, это достаточно просто для рациональных и тригонометрических моделей Калоджеро-Мозера и открытой цепочки Тоды. В этих случаях решение задачи Коши сводится к чисто алгебраическим вычислениям без квадратур [1], [2], [4], [5].

Такие системы отличаются простой зависимостью оператора Лакса от спектрального параметра. При этом соответствующие спектральные кривые - это рациональные кривые с особенностями. При построении решений для таких систем методом проектирования зависимость от спектрального параметра становится несущественной.

Сушествуют также алгебро-геометрические методы построения решений, основанные на методе конечно-зонного интегрирования (см., например, обзор [9]).Для упомянутых систем алгебро-геометрические конструкции были предложены сравнительно недавно [10], [11]. Алгебро-геометрическая процедура выглядит более сложной, чем метод проектирования, но ведет к аналогичным ответам. Для более сложных матриц Лакса вычисления калибровочно-ковариантных величин, необходимых в методе проектирования, является крайне нетривиальной задачей, и алгебро-геометрические методы оказываются предпочтительными.

Здесь мы сравниваем два этих подхода для промежуточной системы - периодической цепочки Тоды, в которой зависимость от спектрального параметра оказывается не слишком сложной. Метод проектирования может быть сформулирован в терминах задачи Римана-Гильберта, т.е. разложения Гаусса бесконечных матриц. С другой стороны, сушествует полученная в [11], [12] алгебро-геометрическим методом формула Кричевера. Она дает явный ответ для периодической цепочки Тоды в терминах тета-функций Римана.

\section{2. ПЕРИОДИЧЕСКАЯ ЦЕПОЧКА ТОДЫ КАК РЕДУЦИРОВАННАЯ СИСТЕМА}

2.1. Определение периодической цепочки Тоды. Иерархия периодической цепочки Тоды описывает одномерную систему $N$ частиц с координатами $\mathbf{u}=\left(u_{1}, \ldots\right.$ $\left.\ldots, u_{N}\right)$ и импульсами $\mathbf{v}=\left(v_{1}, \ldots, v_{N}\right)$. Это вполне интегрируемая система с канонической симплектической формой

$$
\omega=(\delta \mathbf{v}, \delta \mathbf{u})=\sum_{j=1}^{N} \delta v_{j} \delta u_{j}
$$

на фазовом пространстве цепочки Тоды

$$
\mathcal{R}^{T}=(\mathbf{v}, \mathbf{u}), \quad \sum_{j=1}^{N} v_{j}=0, \quad \sum_{j=1}^{N} u_{j}=0 .
$$


Гамильтониан второго порядка по импульсам имеет вид

$$
H_{2}=\frac{1}{2} \sum_{j=1}^{N} v_{j}^{2}+\sum_{j=1}^{N-1} e^{u_{j}-u_{j-1}}+e^{u_{N}-u_{1}}
$$

Существуют еще $N-2$ коммутирующих гамильтонианов, что обеспечивает полную интегрируемость системы. Они имеют вид

$$
\begin{gathered}
H_{3}=\frac{1}{3} \sum_{j=1}^{N} v_{j}^{3}+v_{j}\left(e^{u_{j+1}-u_{j}}+e^{u_{j}-u_{j-1}}\right) \quad\left(u_{N+1}=u_{1}\right) \\
\ldots \ldots \ldots \ldots \ldots \ldots \cdots \cdots \\
H_{k}=\frac{1}{k} \sum_{j=1}^{N} v_{j}^{k}+\cdots
\end{gathered}
$$

Если последний член в потенциале (2.2) отсутствует, то соответствующая система носит название непериодической цепочки Тоды.

Уравнения движения могут быть представлены в форме Лакса:

$$
\partial_{j} L=\left[L, M_{j}\right], \quad \partial_{j}=\partial_{t_{j}},
$$

где $t_{j}$ - время, отвечающее эволюции по отношению к гамильтониану $H_{j}$ и $L=L(\mathbf{v}, \mathbf{u})$, $M_{j}(\mathbf{v}, \mathbf{u})$ - матрищы $N$-го порядка, зависящие от динамических переменных (см. ниже).

2.2. Процедура редукции. Мы описываем здесь процедуру редукции для периодической цепочки Тоды. Периодическая цепочка Тоды получается как результат симплектической редукции из свободной иерархии, заданной на кокасательном расслоении к группе петель $L(\mathrm{SL}(N, \mathbb{C}))$. Группа петель $L(\mathrm{SL}(N, \mathbb{C}))$ есть пространство отображений $\mathbb{C}^{*} \rightarrow \mathrm{SL}(N, \mathbb{C}):$

$$
L(\mathrm{SL}(N, \mathbb{C}))=\left\{g(z) \mid g(z)=\sum_{a=-\infty}^{\infty} g_{a} z^{a}, \quad g_{a} \in \mathrm{SL}(N, \mathbb{C}), \quad z \in \mathbb{C}^{*}\right\}
$$

где только конечное число матричных коэффициентов $g_{a}$ отлично от нуля. Пусть $\Phi$ есть 1 -форма на $\mathbb{C}^{*}$, принимающая значения в группе $\operatorname{Lie}^{*}(\operatorname{sl}(N, \mathbb{C}))$, изоморфной $\operatorname{sl}(N, \mathbb{C})$. Тогда пара $(\Phi, g)$ параметризует кокасательное расслоение $T^{*} L(\operatorname{SL}(N, \mathbb{C}))$. Каноническая симплектическая форма на $T^{*} L(\mathrm{SL}(N, \mathbb{C}))$ есть

$$
\omega=\frac{1}{2 \pi i} \oint_{|z|=1} \operatorname{tr}\left(\delta\left(\Phi g^{-1} \delta g\right)\right) \frac{d z}{z}
$$

Она инвариантна по отношению к левым и правым умножениям

$$
\begin{array}{lll}
g \rightarrow g f^{\mathrm{R}}, & \Phi \rightarrow\left(f^{\mathrm{R}}\right)^{-1} \Phi f^{\mathrm{R}}, & f^{\mathrm{R}} \in L(\mathrm{SL}(N, \mathbb{C})) \\
g \rightarrow f^{\mathrm{L}} g, & \Phi \rightarrow \Phi, & f^{\mathrm{L}} \in L(\mathrm{SL}(N, \mathbb{C})) .
\end{array}
$$


На $T^{*} L(\mathrm{SL}(N, \mathbb{C}))$ существуют калибровочно-инвариантные гамильтонианы

$$
H_{k}=\frac{1}{k} \oint_{|z|=1} \operatorname{tr}\left(\Phi^{k}\right) \frac{d z}{z}, \quad k=2, \ldots, N,
$$

которые, как легко видеть, находятся в инволюции.

Выпишем уравнения эволюции гамильтоновой иерархии (2.4), (2.7):

$$
\partial_{j} \Phi=0, \quad g^{-1} \partial_{j} g=\Phi^{j-1}, \quad \partial_{j}=\partial_{t_{j}},
$$

где времена $t_{j}$ отвечают гамильтонианам $H_{j}$. Уравнения интегрируются очевидным образом:

$$
\Phi\left(t_{j}\right)=\Phi_{0}, \quad g\left(t_{2}, \ldots, t_{N}\right)=g_{0} \exp \left(\sum_{j=2}^{N} t_{j} \Phi_{0}^{j-1}\right) .
$$

Рассмотрим в $\operatorname{SL}(N, \mathbb{C})$ нильпотентные подгруппы $N^{(+)}\left(N^{(-)}\right)$верхне- (нижне-) треугольных матриц с единицами по диагонали. Определим подгруппу $L^{(-)}$в $L(\operatorname{SL}(N, \mathbb{C}))$ кaK

$$
L^{(-)}=\left\{\left.g(z)\right|_{z \rightarrow \infty} \rightarrow n_{-} \in N^{(-)}\right\}
$$

и $L^{(+)}$как

$$
L^{(+)}=\left\{\left.g(z)\right|_{z \rightarrow 0} \rightarrow n_{+} \in N^{(+)}\right\} .
$$

Так как правое действие $(2.5)$ группы $L^{(-)}$есть каноническое преобразование формы $\omega(2.4)$, то можно написать соответствуюший гамильтониан, генерируюший правое умножение (2.5),

$$
\operatorname{tr}(\Phi \epsilon), \quad \epsilon \in \operatorname{Lie}\left(L^{(-)}\right)
$$

и отображение момента

$$
\begin{aligned}
& \mu_{\mathrm{R}}: T^{*} L(\operatorname{SL}(N, \mathbb{C})) \rightarrow \operatorname{Lie}^{*}\left(L^{(-)}\right) \sim \operatorname{Lie}\left(L^{(+)}\right), \\
& \mu_{\mathrm{R}}(\Phi, g)=\left.\operatorname{Pr}\right|_{\operatorname{Lie}\left(L^{(+)}\right)}(\Phi) .
\end{aligned}
$$

Аналогично левое действие (2.6) группы $L^{(+)}$задается гамильтонианом

$$
\operatorname{tr}\left(g \Phi g^{-1} \epsilon\right), \quad \epsilon \in \operatorname{Lie}\left(L^{(+)}\right) .
$$

Ему отвечает отображение момента

$$
\mu_{\mathrm{L}}(\Phi, g)=\left.\operatorname{Pr}\right|_{\operatorname{Lie}\left(L^{(-)}\right)}\left(g \Phi g^{-1}\right) .
$$

Мы выбираем следующие величины моментов:

$$
\mu_{\mathrm{R}}=\delta_{j, k-1}+z \delta_{j, N} \delta_{k, 1}, \quad \mu_{\mathrm{L}}=\delta_{j, k+1}+z^{-1} \delta_{j, 1} \delta_{k, N},
$$

и приходим к уравнениям моментов

$$
\begin{aligned}
\left.\operatorname{Pr}\right|_{\operatorname{Lie}\left(L^{(+)}\right)}(\Phi) & =\delta_{j, k-1}+z \delta_{j, N} \delta_{k, 1}, \\
\left.\operatorname{Pr}\right|_{\operatorname{Lie}\left(L^{(-)}\right)}\left(g \Phi g^{-1}\right) & =\delta_{j, k+1}+z^{-1} \delta_{j, 1} \delta_{k, N} .
\end{aligned}
$$


Это минимальный выбор, т.к. вся подгруппа $L^{(-)}$сохраняет $\mu_{\mathrm{R}}$, а подгруппа $L^{(+)}$сохраняет $\mu_{\mathrm{L}}$.

Для открытого плотного подмножества в $L(\mathrm{SL}(N, \mathbb{C}))$ справедливо разложение Бирхгофа [13]

$$
g=r_{+} h r_{-}, \quad r_{+} \in L^{(+)}, \quad r_{-} \in L^{(-)}, \quad h \in D \subset \mathrm{SL}(N, \mathbb{C}),
$$

где $D$ - диагональная (картановская) подгруппа группы $\operatorname{SL}(N, \mathbb{C})$. Пусть $U_{+}, U_{-}$ окрестности точек $z=0$ и $z=\infty$ такие, что $\mathbb{C} P^{1}=U_{+} \cup U_{-}$. Матричная функция $g(z)$ есть функция переклейки тривиального голоморфного $\mathrm{SL}(N, \mathbb{C})$-расслоения над $\mathbb{C} P^{1}$. При этом разрешены левые умножения $g(z)$ на голоморфные на $U_{+}$матричные функции, принадлежащие $L^{(+)}$. Аналогично разрешенные правые множители принадлежат $L^{(-)}$. Условия $(2.10),(2.11)$ означают, что расслоение имеет фиксированную квазипараболическую структуру в точках 0 и $\infty$. Пространство модулей таких расслоений описывается элементами картановской подгрупшы $D$.

Как мы уже упоминали, матрицы из $L^{(-)}$и $L^{(+)}$, диагонализующие $g$, сохраняют момент (2.14). Зафиксируем калибровку в виде

$$
h=\operatorname{diag}\left(e^{u_{1}}, \ldots, e^{u_{N}}\right), \quad \sum_{j} u_{j}=0 .
$$

При этом матрица $\Phi$ преобразуется как

$$
\Phi=r_{-}^{-1} L r_{-} .
$$

Так как при калибровочных преобразованиях момент (2.14) сохраняется, то можно подставить $h$ и $L$ в уравнения связи $(2.15),(2.16)$ :

$$
\begin{aligned}
\left.\operatorname{Pr}\right|_{\operatorname{Lie}\left(L^{(+)}\right)}(L) & =\delta_{j, k-1}+z \delta_{j, N} \delta_{k, 1}, \\
\left.\operatorname{Pr}\right|_{\operatorname{Lie}\left(L^{(-)}\right)}\left(h L h^{-1}\right) & =\delta_{j, k+1}+z^{-1} \delta_{j, 1} \delta_{k, N} .
\end{aligned}
$$

Тогда определится вся матрица $L$, кроме главной диагонали. Диагональные матричные элементы можно рассматривать как координаты в пространстве модулей решений уравнений связи

$$
\operatorname{diag}(L)=\operatorname{diag}\left(v_{1}, \ldots, v_{N}\right), \quad \sum_{j} v_{j}=0 .
$$

Окончательно мы придем к решению

$$
\begin{array}{r}
L_{j, k}=v_{j} \delta_{j, k}+\delta_{j, k-1}+e^{u_{j}-u_{j-1}} \delta_{j, k+1}+ \\
+z \delta_{j, N} \delta_{k, 1}+z^{-1} e^{u_{N}-u_{1}} \delta_{j, 1} \delta_{k, N} .
\end{array}
$$

Таким образом, редуцированное фазовое пространство описывается парой матриц

$$
\mathcal{R}^{\mathrm{red}}=T^{*} L(\mathrm{SL}(N, \mathbb{C})) / / L^{(+)} \oplus L^{(-)} \sim(L, h) .
$$


Из (2.18) и (2.20) следует, что форма $(2.4)$ на $\mathcal{R}^{\text {red }}$ совпадает с канонической формой (2.1) цепочки Тоды. Гамильтонианы (2.7), будучи ограниченными на $\mathcal{R}^{\text {red }}$, порождают гамильтонианы иерархии цепочки Тоды. Они находятся в инволюции, так как исходные гамильтонианы коммутируют. Следовательно, редуцированная система есть иерархия цепочки Тоды.

Подставляя (2.19) в первое уравнение (2.18), мы заключаем, что матрица $L$ удовлетворяет уравнению Лакса (2.3), где

$$
M_{j}=\partial_{j} r_{-} r_{-}^{-1}
$$

Матришы $M_{j}$ могут быть найдены с помошью второго уравнения (2.8). Оно дает соотношение между $L$ и $M_{j}$ :

$$
h^{-1} r_{+}^{-1} \partial_{j} r_{+} h+h^{-1} \partial_{j} h+M_{j}=L^{j-1} .
$$

Так как $r_{-} \in L^{(-)}$, то из (2.21) следует, что $M_{j} \in \operatorname{Lie}\left(L^{(-)}\right)$и поэтому

$$
M_{j}=\left.\operatorname{Pr}\right|_{\operatorname{Lie}\left(L^{(-)}\right)} L^{j-1} .
$$

В частности,

$$
M_{2}=e^{u_{j}-u_{j-1}} \delta_{j, k+1}+z^{-1} e^{u_{1}-u_{N}} \delta_{j, 1} \delta_{k, N} .
$$

Матрицы $L(2.20)$ и $M_{j}(2.22)$ образуют пары Лакса для иерархии цепочки Тоды.

Открытая цепочка Тоды может быть получена, если исходить из конечномерной группы $\mathrm{SL}(N, \mathbb{C})$ вместо группы петель и использовать разложение Гаусса вместо разложения Бирхгофа. Соответствуюший оператор Лакса задается формулой (2.20), в которой два последних зависяших от спектрального параметра члена опущены. Операторы $M_{j}$ аналогичным (2.22) образом связаны с модифицированным оператором $L$.

Подобный вывод может быть повторен для произвольной простой аффинной алгебры $L(G)$.

\section{3. ПОСТРОЕНИЕ РЕШЕНИЙ С ПОМОШЬЮ МЕТОДА ПРОЕКТИРОВАНИЯ}

3.1. Линеаризация. Пусть $\mathbf{u}^{0}=\left(u_{1}^{0}, \ldots, u_{N}^{0}\right)$ и $\mathbf{v}^{0}=\left(v_{1}^{0}, \ldots, v_{N}^{0}\right)$ - данные Коши для иерархии цепочки Тоды. В начальные моменты времен $t_{j}=0, j=2, \ldots, N$, положим

$$
\begin{aligned}
\left.g\left(t_{2}, \ldots, t_{N}\right)\right|_{t_{j}=0} & =\left.h\left(t_{2}, \ldots, t_{N}\right)\right|_{t_{j}=0}=h_{0}, \\
\left.\Phi\left(t_{2}, \ldots, t_{N}\right)\right|_{t_{j}=0} & =\left.L\left(t_{2}, \ldots, t_{N}\right)\right|_{t_{j}=0}=L_{0} .
\end{aligned}
$$

Тогда в соответствии с (2.9) эволюция в исходном пространстве линейна:

$$
g(\mathbf{t})=X\left(\mathbf{v}^{0}, \mathbf{u}^{0}, \mathbf{t}, z\right)
$$


где $\mathbf{t}=\left(t_{2}, \ldots, t_{n}\right)$ и

$$
X\left(\mathbf{v}^{0}, \mathbf{u}^{0}, \mathbf{t}, z\right)=h_{0} \exp \left(\sum_{j=2}^{N} t_{j} L_{0}^{j-1}(z)\right)
$$

Координаты Тоды получаются из диагональной части разложения Бирхгофа (2.17):

$$
h(\mathbf{t})=r_{+} X\left(\mathbf{v}^{0}, \mathbf{u}^{0}, \mathbf{t}, z\right) r_{-} \rightarrow \mathbf{u}(\mathbf{t})=\ln \left(r_{+} X r_{-}\right)
$$

Первый шаг в процедуре проектирования есть вычисление по данным Коши (3.1) матрицы $X\left(\mathbf{v}^{0}, \mathbf{u}^{0}, \mathbf{t}, z\right)$. Затем с помошью формулы Бирхгофа надо диагонализовать $X\left(\mathbf{v}^{0}, \mathbf{u}^{0}, \mathbf{t}, z\right)$. Это можно осушествить двумя способами - либо решая задачу Римана, либо с помощью разложения Гаусса в GL( $\infty)$. Прежде чем описать два эти метода, мы решим задачу Коши в более простом случае - для открытой цепочки Тоды.

3.2. Решения в открытой цепочке Тоды. В случае открытой цепочки Тоды, как мы уже объясняли, матрица $X\left(\mathbf{v}^{0}, \mathbf{u}^{0}, \mathbf{t}\right)$ не зависит от спектрального параметра и в формуле (3.2) $r_{+}$и $r_{-}-$постоянные треугольные матрицы: $r_{+} \in N^{(+)}, r_{-} \in N^{(-)}$. Диагональные элементы $h(\mathbf{t})=\operatorname{diag}\left(h_{1}(\mathbf{t}), \ldots, h_{N}(\mathbf{t})\right) \in D$ извлекаются из разложения Гаусса

$$
\operatorname{SL}(N, \mathbb{C})=N^{(+)} D N^{(-)} .
$$

На самом деле разложение Гаусса справедливо в открытом плотном подмножестве $\operatorname{SL}(N, \mathbb{C})$. Пусть $\Delta_{j}(g)$ есть главный нижний минор матрицы $g \in \operatorname{SL}(N, \mathbb{C})$ порядка $j$ $\left(j=1, \ldots, N, \Delta_{N}=1\right)$. Тогда это множество диагональных элементов характеризуется условиями $\Delta_{j}(g) \neq 0, j=1, \ldots, N-1$. Более того, миноры $\Delta_{j}(g)$ инвариантны по отношению к умножению $g$ справа на матрицы из $N^{(+)}$и слева на матрицы из $N^{(-)}$. Отношение миноров определяет диагональные элементы в разложении Гаусса:

$$
h_{j}=\frac{\Delta_{N-j+1}\left(X\left(\mathbf{v}^{0}, \mathbf{u}^{0}, \mathbf{t}\right)\right)}{\Delta_{N-j}\left(X\left(\mathbf{v}^{0}, \mathbf{u}^{0}, \mathbf{t}\right)\right)} .
$$

При этом мы получим

$$
u_{j}=\ln \frac{\Delta_{N-j+1}\left(X\left(\mathbf{v}^{0}, \mathbf{u}^{0}, \mathbf{t}\right)\right)}{\Delta_{N-j}\left(X\left(\mathbf{v}^{0}, \mathbf{u}^{0}, \mathbf{t}\right)\right)}
$$

Из (3.1) следует, в частности, что миноры $\Delta_{j}\left(e^{X\left(\mathbf{v}^{0}, \mathbf{u}^{0}, \mathbf{t}\right)}\right)$ зависят от времени как экспоненциальные полиномы.

3.3. Нахождение решений с помощью задачи Римана. Перепишем разложение Бирхгофа (2.17) в виде

$$
g=b \tilde{r}_{-}^{-1},
$$

где $\tilde{r}_{-}^{-1}$ - матрица, голоморфная вне контура $|z|=1$ и удовлетворяющая нормировочному условию

$$
\lim _{z \rightarrow \infty} \tilde{r}_{-} \rightarrow \text { Id }
$$


Иными словами, $\tilde{r}_{-}=\mathrm{Id}+\sum_{j>0} r_{j} z^{-j}$. Матрица $b$ голоморфна внутри контура $|z|=1$. Наша конечная цель - найти постоянную диагональную компоненту $b$. Она находится с помошью решения задачи Римана. А именно, сначала найдем $\tilde{r}_{-}(z)$-компоненту матрицы $g(z)$. Для этого перепишем (3.4) в виде

$$
b-\mathrm{Id}=(g-\mathrm{Id}) \tilde{r}_{-}+\tilde{r}_{-}-\mathrm{Id} .
$$

Так как $b-\mathrm{Id}$ - голоморфная матрица в $U_{+}$, то $\tilde{r}_{-}$удовлетворяет матричному сингулярному интегральному уравнению

$$
\frac{1}{2 \pi i} \oint_{|w|=1} \frac{(g(w)-\mathrm{Id}) \tilde{r}_{-}(w)}{z-w}+\tilde{r}_{-}(z)-\mathrm{Id}=0
$$

с нормировкой (3.5). Зная решение $\tilde{r}_{-}(z)$, можно найти $b$. Пусть $b=b_{0}+\sum_{j>0} b_{j} z^{j}$, тогда $b_{0}=\lim _{z \rightarrow 0}\left(g \tilde{r}_{-}\right)$. Затем, чтобы найти $h=\operatorname{diag}\left(e^{u_{1}}, \ldots, e^{u_{N}}\right)$, в (3.2) можно воспользоваться разложением Гаусса для $b_{0}$, как уже было сделано в открытой цепочке Тоды (3.3).

Подставляя $g(w)=X$ из (3.1) в уравнение $(3.6)$, мы найдем $\tilde{r}_{-}(z)$. Тогда

$$
b\left(\mathbf{v}^{0}, \mathbf{u}^{0}, \mathbf{t}, z\right)=X\left(\mathbf{v}^{0}, \mathbf{u}^{0}, \mathbf{t}, z\right) \tilde{r}_{-}(z)=h(0, \ldots, 0) \exp \left(\sum_{j=2}^{N} t_{j} L_{0}^{j-1}(z)\right) \tilde{r}_{-}(z)
$$

Отсюда окончательно находим решение

$$
u_{j}(\mathbf{t})=\lim _{z \rightarrow 0} \ln \frac{\Delta_{N-j+1}\left(b\left(\mathbf{v}^{0}, \mathbf{u}^{0}, \mathbf{t}, z\right)\right)}{\Delta_{N-j}\left(b\left(\mathbf{v}^{0}, \mathbf{u}^{0}, \mathbf{t}, z\right)\right)} .
$$

3.4. Нахождение решений с помощью вложения в $\mathrm{GL}(\infty)$. Мы определим $\mathrm{GL}(\infty)$ в алгебраических терминах. Детальный анализ структуры этой группы изложен в [13], [14].

Пусть $\mathcal{H}$ - гильбертово пространство функций на $S^{1}$. Группа GL( $\left.\infty\right)$ есть подгруппа ограниченных операторов, действующих в $\mathcal{H}$, таких, что матричные элементы $M_{j, k}$ группы GL $(\infty)$ в базисе $\Phi$ урье $e^{i m \varphi}$ обращаются в нуль при больших значениях $|j-k|$.

Группа $L(\mathrm{SL}(N, \mathbb{C}))$ может быть отображена в $\mathrm{GL}(\infty)$. Для этого рассмотрим в $\mathrm{GL}(\infty)$ подгруппу периодических матриц

$$
\operatorname{GL}_{\text {per }}(\infty)=\left\{M_{j+m N, k+m N}=M_{j, k}\right\}
$$

Пусть

$$
g(z) \in L(\mathrm{SL}(N, \mathbb{C})), \quad g_{j k}(z)=\sum_{n}\left(g_{j k}\right)_{n} z^{n} \quad(j, k=1, \ldots, N) .
$$

Тогда образ $g(z)$ матрицы $M \in \mathrm{GL}_{\mathrm{per}}(\infty)$ определяется как

$$
M_{s, t}=\left(g_{j k}\right)_{n}, \quad s=j+m N, \quad t=k+(m+n) N, \quad s, t, m \in \mathbb{Z} .
$$


При этом подгруппа $L^{(-)}$, определенная в $(2.10)$, отображается в нижнетреугольные матрицы из $\mathrm{GL}_{\mathrm{per}}(\infty)$, а $L^{(+)}(2.11)$ - в верхнетреугольные матрищы. Таким образом, разложение Бирхгофа (2.17) переходит в разложение Гаусса $\mathrm{GL}_{\text {per }}(\infty)$. Постоянные диагональные петли отображаются в диагональные периодические матрицы.

Пусть образ $X\left(\mathbf{v}^{0}, \mathbf{u}^{0}, \mathbf{t}, z\right)$ (из $\left.(3.1)\right)$ в $\mathrm{GL}_{\text {per }}(\infty)$ есть $\widetilde{X}\left(\mathbf{v}^{0}, \mathbf{u}^{0}, \mathbf{t}\right), \operatorname{det}_{j}(\widetilde{X})$ - детерминанты полубесконечных матриц $\widetilde{X}_{m, n}, m, n \in \mathbb{Z}, m, n \leqslant j$. Такие детерминанты плохо определены, но их отношения конечны. Из (3.2) следует, что решения могут быть выражены через отношения

$$
u_{j}(\mathbf{t})=\ln \frac{\Delta_{j+1}(\widetilde{X})}{\Delta_{j}(\widetilde{X})} .
$$

Это выражение связано с $\tau$-функциями двумерной иерархии Тоды, введенной в [14] (см. ниже).

Мы с помощью метода проектирования получили два неявных представления - (3.7) и (3.8) - решений задачи Коши. В следуюшем разделе мы представим их явно в терминах тета-функций Римана.

\section{4. АЛГЕБРО-ГЕОМЕТРИЧЕСКИЕ ВЫЧИСЛЕНИЯ}

При описании интегрируемых моделей естественным образом появляются голоморфные векторные расслоения. Один из классов таких расслоений, связанных с периодической цепочкой Тоды, был описан выше. Как мы видели, координатное пространство связано с модулями голоморфных расслоений. Сами расслоения могут быть восстановлены из спектральных характеристик оператора Лакса. Это дает возможность строить решения по спектральным данным (см., например, обзор [9]).

Здесь мы воспроизведем решение задачи Коши для периодической цепочки Тоды, построенное с помощью этого подхода Кричевером [11], [12].

Пусть $\mathcal{C}_{N}$ - спектральная кривая, определенная с помощью характеристического полинома $C_{N}(\lambda)$ матрицы Лакса $L$ :

$$
C_{N}(\lambda, z)=0, \quad C_{N}(\lambda, z)=\operatorname{det}(L(z)-\lambda \cdot \operatorname{Id}) .
$$

Из (2.19) следует, что (4.1) - калибровочно-инвариантное уравнение. Из-за того что оператор Лакса имеет специальный вид (2.20), спектральная кривая $C_{N}$ является гиперэллиптической кривой

$$
C_{N}(z, \lambda)=z+z^{-1}+R_{N}(\lambda), \quad R_{N}(\lambda)=\lambda^{N}+\sum_{k=2}^{N} I_{k} \lambda^{N-k} .
$$

Здесь коэффициенты $I_{k}$ - это интегралы движения других типов. Кривая имеет род $N-1$. Она представляет двулистное накрытие $\lambda$-плоскости, где корни $\lambda_{1}, \ldots, \lambda_{2 N}$ уравнения $R_{N}(\lambda)=4$ являются точками ветвления.

Пространство голоморфных дифференциалов на $\mathcal{C}_{N}$ порождается $\lambda^{j} d \lambda / d y, j=0, \ldots$ $\ldots, N-2$, где $y=z+(1 / 2) R_{N}(\lambda)$. Составим их линейную комбинацию

$$
\omega_{i}=\sum_{j=0}^{N-2} a_{i j} \lambda^{j} \frac{d \lambda}{d y}, \quad i=1, \ldots, N-1,
$$


такую, что

$$
2 \int_{\lambda_{2 k-1}}^{\lambda_{2 k}} \omega_{i}=\delta_{i k}, \quad k=1, \ldots, N-1,
$$

где интеграл берется по верхнему листу. Дифференциалы $\omega_{i}$ суть нормированные дифоференциалы первого рода. Матрица $B$ их $b$-периодов

$$
B_{j k}=2 \int_{\lambda_{2 j}}^{\lambda_{2 N}} \omega_{k}
$$

позволяет определить якобиан $\mathcal{J}\left(\mathcal{C}_{N}\right)$ кривой $\mathcal{C}_{N}$ :

$$
\begin{gathered}
\mathcal{J}\left(\mathcal{C}_{N}\right)=\mathbb{C}^{N-1} /\left(\bigoplus_{j} \mathbb{Z} e_{j}\right) \oplus\left(\bigoplus_{k} \mathbb{Z} B_{k}\right), \\
e_{j}=\left(0, \ldots,{ }_{j}, 0 \ldots, 0\right), \quad B_{k}=\left(B_{1 k}, \ldots, B_{N-1, k}\right) .
\end{gathered}
$$

Вектор $\left(\omega_{1}, \ldots, \omega_{N-1}\right)$ с помошью формулы

$$
\omega_{k}(\gamma)=\int_{\lambda_{2 N}}^{\gamma} \omega_{k}
$$

задает отображение Абеля $\mathcal{C}_{N} \rightarrow \mathcal{J}\left(\mathcal{C}_{N}\right)$.

Сушествует изоморфизм между подмногообразием $M_{f}$ в фазовом пространстве $\mathcal{R}^{T}$ с фиксированным уровнем интегралов движения и якобианом $\mathcal{J}\left(\mathcal{C}_{N}\right)$. Таким образом, якобиан играет роль тора Лиувилля. По точке фазового пространства $(\mathbf{v}, \mathbf{u}) \in \mathcal{R}^{T}$ определим $N-1$ точку $\gamma_{j}=\left(\mu_{j}, z_{j}\right)$ на спектральной кривой $\mathcal{C}_{N}$. Здесь $\mu_{j}$ есть корень характеристического полинома левого главного минора порядка $N-1$ матрицы $L$ из (2.20), а $z_{j}$ есть корень полинома (4.1) при $\lambda=\mu_{j}$, для которого правый главный минор $L$ невырожденный. Изоморфизм $M_{f} \rightarrow \mathcal{J}\left(\mathcal{C}_{N}\right)$ определяется с помошью вектора $\mathbf{w}=\left(w_{1}, \ldots, w_{N_{1}}\right):$

$$
w_{k}=-\sum_{n=1}^{N-1} \omega_{k}\left(\gamma_{n}\right)+\frac{1}{2}-\frac{1}{2} \sum_{j \neq k} \int_{\lambda_{2 j-1}}^{\lambda_{2 j}} \omega\left(\int_{\lambda_{2 N}}^{t} \omega_{k}\right) d t .
$$

Рассмотрим дифференциалы

$$
s^{1}=\frac{\lambda^{N-1} d \lambda}{y(z, \lambda)}+\sum_{i=0}^{N-2} \alpha_{i} \frac{\lambda^{i} d \lambda}{y(z, \lambda)}, \quad s^{2}=\frac{\lambda^{N} d \lambda}{y(z, \lambda)}+\sum_{i=0}^{N-2} \beta_{i} \frac{\lambda^{i} d \lambda}{y(z, \lambda)},
$$

нормализованные следуюшим образом:

$$
\int_{\lambda_{2 j-1}}^{\lambda_{2 j}} s^{1,2}=0 .
$$


Пусть $S^{1}, S^{2}, Z^{ \pm}$- векторы с координатами

$$
S_{j}^{1,2}=\frac{1}{i \pi} \int_{\lambda_{2 j}}^{\lambda_{2 N}} s^{1,2}, \quad Z_{j}^{ \pm}=\int_{\lambda_{2 N}}^{ \pm \lambda_{2 N}} \omega_{j} .
$$

В первых двух интегралах интегрирование производится по верхнему листу спектральной кривой $\mathcal{C}_{N}$. В последних интегралах интегрирование производится по верхнему и нижнему листам.

Теперь мы в состоянии определить решение задачи Коши при эволюции в соответствии с квадратичным гамильтонианом $H_{2}$. Начальные данные $\left(\mathbf{v}_{0}, \mathbf{u}_{0}\right)$ определяют спектральную кривую, матрицу периодов $B$, векторы $S^{1,2}, Z^{ \pm}$и $\mathbf{w}^{0}=\mathbf{w}\left(\mathbf{v}_{0}, \mathbf{u}_{0}\right)$ из (4.2). Пусть $\Theta(\mathbf{x})$ - тета-функция Римана

$$
\Theta(\mathbf{x})=\sum_{\mathbf{m} \in \mathbb{Z}^{N-1}} \exp \{i \pi(B \mathbf{m}, \mathbf{m})+2 i \pi(\mathbf{m x})\}, \quad B=B_{j k} .
$$

Тогда решение имеет вид

$$
u_{j}(t)=\ln \frac{\Theta\left(Z^{+}+\frac{1}{2} S^{2} t+S^{1}(j-1)+\mathbf{w}^{0}\right) \Theta\left(Z^{-}+\mathbf{w}^{0}\right)}{\Theta\left(Z^{-}+\frac{1}{2} S^{2} t+S^{1}(j-1)+\mathbf{w}^{0}\right) \Theta\left(Z^{+}+\mathbf{w}^{0}\right)}+(j-1) C+u_{j}(0),
$$

где

$$
Z^{ \pm}=\left(Z_{1}^{ \pm}, \ldots, Z_{N}^{ \pm}\right), \quad S^{1,2}=\left(S_{1}^{1,2}, \ldots, S_{N}^{1,2}\right)
$$

и константа $C$ выбрана так, что $u_{N+1}=u_{1}$.

Эту формулу можно сравнить с двумя выражениями (3.7) и (3.8), полученными ранее с помошью метода проектирования. Можно предположить, что отношения построенных тета-функций совпадают с отношением регуляризованных детерминантов полубесконечных матриц $\widetilde{X}(3.8)$.

\section{5. ДРУГИЕ КОНСТРУКЦИИ}

Существуют два других подхода, позволяющих описывать решения периодической цепочки Тоды. Хотя исходно они отличаются от метода проектирования, в конечном итоге они формулируются в терминах задачи Римана.

5.1. Тау-функции периодической цепочки Тоды. В двух статьях [14], [15] рассматривалась иерархия теории поля Тоды с бесконечным числом полей. Иерархия была сформулирована в терминах $\mathrm{GL}(\infty)$. Она зависела от двух бесконечных множеств времен $\mathbf{x}=\left(x_{1}, x_{2}, \ldots\right), \mathbf{y}=\left(y_{1}, y_{2}, \ldots\right)$. Ее одномерный вариант зависит только от комбинации $\tilde{t}_{2}=\left(x_{1}-y_{1}\right) / 2, \tilde{t}_{3}=\left(x_{2}-y_{2}\right) / 2, \ldots$ В этом случае бесконечная иерархия может быть сведена к конечному числу уравнений Лакса. Времена $\tilde{t}_{j}$ отвечают операторам $\widetilde{M}_{j}$, отличным от $(2.22)$. В терминах [14]:

$$
L=B_{1}+C_{1}, \quad \widetilde{M}_{j+1}=B_{j}-C_{j},
$$


где $C_{1}=M_{2}=L_{-}, B_{1}=L_{+}+\operatorname{diag} L$. Рассмотрим следуюшую линейную задачу:

$$
\begin{gathered}
L V(\mathbf{t})=\left(\mu_{\mathrm{L}}+\mu_{\mathrm{R}}\right) V(\mathbf{t}), \\
\partial_{j} V(\mathbf{t})=\widetilde{M}_{j} V(\mathbf{t}), \quad j=2, \ldots, N, \quad \partial_{j}=\partial_{\tilde{t}_{j}},
\end{gathered}
$$

где матрицы $\mu_{\mathrm{L}}$ и $\mu_{\mathrm{R}}-$ моменты $(2.14)$. Уравнения Лакса для $L$ и $\widetilde{M}_{j}-$ это условия совместности для данной двумерной системы. Матричная функция $V(\mathbf{t})$ может рассматриваться как матричная волновая функция, "одеваюшая" операторы $\mu_{\mathrm{L}}+\mu_{\mathrm{R}}$ и $\partial_{j}$. Матричные элементы для $\mathbf{y}$ могут быть выражены через отношения функций $\tau(\mathbf{x}, \mathbf{y})$, определенных в [14] для всей двумерной иерархии. Функции одномерной периодической цепочки Тоды образуют специальный подкласс двумерных тау-функций, которые не зависят от времен $\mathbf{x}+\mathbf{y}$ и удовлетворяют определенным периодическим условиям.

Решение задачи Коши в этих терминах, предложенное в [15], может быть построено следуюшим образом. Прежде всего, по начальным данным строится волновая функция $V^{0}=V(\mathbf{t}=0)$ из (5.1). Сушествует представление для тау-функций, определенных при произвольных значениях времен и зависяших от матричных элементов $V^{0}$. Волновые функции $V(\mathbf{t})$, в свою очередь, выражаются через отношения тау-функций $\tau_{j}(\mathbf{t})$. Последний шаг - решение обратной задачи в (5.1). Итак, решение задачи Коши может быть проиллюстрировано цепочкой шагов

$$
\left(\mathbf{v}^{0}, \mathbf{u}^{0}\right) \rightarrow\left(L^{0}, \widetilde{M}_{j}^{0}\right) \rightarrow V^{0} \rightarrow \tau(\mathbf{t}) \rightarrow V(\mathbf{t}) \rightarrow(L(\mathbf{t}))
$$

В этой схеме задача Римана возникает на этапе построения тау-функций.

5.2. Метод $R$-матрицы. Из определения подгрупп $L^{(-)}, L^{(+)}(2.10),(2.11)$ следует, что сушествует разложение

$$
\operatorname{Lie}(L(\operatorname{SL}(N, \mathbb{C})))=\operatorname{Lie}\left(L^{(+)}\right) \oplus \mathcal{H} \oplus \operatorname{Lie}\left(L^{(-)}\right)=\operatorname{Lie}\left(B^{(+)}\right) \oplus \operatorname{Lie}\left(L^{(-)}\right),
$$

где $\mathcal{H}$ - подалгебра диагональных матриц в $\operatorname{sl}(N, \mathbb{C})$ и $\operatorname{Lie}\left(B^{(+)}\right)$- борелевская подалгебра. Определим проекцию

$$
M_{j}^{(+)}=-\operatorname{Pr}_{\operatorname{Lie}\left(B^{(+)}\right)} L^{j-1} .
$$


Тогда

$$
L^{j-1}=-M_{j}^{(+)}+M_{j}^{(-)},
$$

где $M_{j}^{(-)}=M_{j}(2.22)$. Иными словами,

где

$$
M_{j}^{( \pm)}=-\frac{1}{2}(R \pm 1) L^{j-1}
$$

$$
R=\left.\operatorname{Pr}\right|_{\operatorname{Lie}\left(L^{(-)}\right)}-\operatorname{Pr}_{\operatorname{Lie}\left(B^{(+)}\right)}
$$

- классическая $R$-матрица, отвечающая разложению (5.2). Тем самым можно написать пару уравнений Лакса для каждого времени $t_{j}$ :

$$
\partial_{j} L=\left[L, M_{j}^{( \pm)}\right] .
$$

Разложение (5.2) отвечает на уровне алгебр Ли разложению Бирхгофа (3.4). Решения уравнений, отвечающих иерархии уравнений Лакса (5.3), могут быть определены в терминах разложения Бирхгофа. Следовательно, мы снова придем к задаче Римана, которая рассматривалась в разделе 2 .

Для общих уравнений Лакса $\mathbb{C} P^{1}$ связь классических $R$-матриц с задачей Римана и алгебро-геометрическими методами интегрирования обсуждалась в [16].

Благодарности. Я признателен за гостеприимство Институту Математических Исследований им. Исаака Ньютона (Кэмбридж), где эта статья была завершена. Я также благодарен В. Новокшонову за полезные обсуждения. Работа была выполнена при поддержке грантов РФФИ № 00-02-16530, № 96-15-96455 и гранта INTAS № 99-01782.

\section{Список литературы}

[1] M. Olshanetsky, A. Perelomov. Lett. Nouvo Cim. 1976. V. 16. P. 333.

[2] M. Olshanetsky, A. Perelomov. Lett. Nouvo Cim. 1976. V. 17. P. 97.

[3] D. Kazdan, B. Kostant, and S. Sternberg. Commun. Pure Appl. Math. 1978. V. 31. P. 481-507.

[4] M. Olshanetsky, A. Perelomov. Invent. Math. 1979. V. 54. P. 261-269.

[5] B. Kostant. Adv. in Math. 1979. V. 34. P. 195-338.

[6] A. Gorsky, N. Nekrasov. Elliptic Calogero-Moser system from two dimensional current algebra. hep-th/9401021.

[7] N. Nekrasov. Commun. Math. Phys. 1996. V. 180. P. 587-604.

[8] N. Hitchin. Duke Math. J. 1987. V. 54. P. 91-114.

[9] Б. А. Дубровин, И. М. Кричевер, С. П. Новиков. Методы алгебраической и топологической геометрии в современной математической физике 2. Обзоры по математической физике. Т. 3. Ред. С. П. Новиков. 1990.

[10] I. Krichever, O. Babelon, E. Billey, and M. Talon. Spin generalization of the Calogero-Moser system and the Matrix KP equation. hep-th/9411160.

[11] I. Krichever, K. Vaninsky. The periodic and open Toda lattice. hep-th/0010184.

[12] M. Olshanetsky, A. Perelomov. Phys. Rep. 1981. V. 71. P. 313-400.

[13] Э. Прессли, Г. Сигал. Группы петель. М.: Мир, 1990.

[14] K. Ueno, K. Takasaki. Proc. Japan Acad., Ser. A. 1983. V. 59. P. 167-170.

[15] K. Takasaki. Adv. Stud. Pure Math. 1984. V. 4. P. 139-163.

[16] А. Рейман, М. Семенов-Тян-Шанский. Теоретико-групповые методы в теории интегрируемых систем. В сб.: Итоги науки и техники. Сер. Современные проблемы математики. Фундаментальные направления. Т. 16. Динамические системы-7. Ред. Р. В. Гамкрелидзе. М.: ВИНИТИ, 1987. С. 119-194. 